ISSN 1999-4915

www.mdpi.com/journal/viruses

Article

\title{
Genomic Analysis of 15 Human Coronaviruses OC43 (HCoV-OC43s) Circulating in France from 2001 to 2013 Reveals a High Intra-Specific Diversity with New Recombinant Genotypes
}

\author{
Nathalie Kin 1,2,, , , Fabien Miszczak ${ }^{1,2,3, \dagger}$, Wei Lin ${ }^{2, \dagger}$, Meriadeg Ar Gouilh ${ }^{1,2,4, \dagger}$, \\ Astrid Vabret ${ }^{1,2,3, \dagger}$ and Epicorem Consortium ${ }^{2}$
}

1 Normandie Université, 14032 Caen, France; E-Mails: fabienmzz@yahoo.fr (F.M.); meriadeg.le-gouil@pasteur.fr (M.A.G.); a-vabret@chu-caen.fr (A.V.)

2 Université de Caen, Unité de Recherche Risques Microbiens (U2RM), F-14000 Caen, France; E-Mails: wei.lin@hotmail.fr (W.L.); epicorem@pasteur.fr (E.C.)

3 Department of Virology, University Hospital of Caen, F-14033 Caen, France

4 Institut Pasteur, Environment and Infectious Risks Research and Expertise Unit, F-75015 Paris, France

$\dagger$ These authors contributed equally to this work.

* Author to whom correspondence should be addressed; E-Mail: nathalie.kin@unicaen.fr; Tel.: +33-0-2-31-27-25-54.

Academic Editor: Eric O. Freed

Received: 25 March 2015 / Accepted: 4 May 2015 / Published: 7 May 2015

\begin{abstract}
Human coronavirus OC43 (HCoV-OC43) is one of five currently circulating human coronaviruses responsible for respiratory infections. Like all coronaviruses, it is characterized by its genome's high plasticity. The objectives of the current study were to detect genetically distinct genotypes and eventually recombinant genotypes in samples collected in Lower Normandy between 2001 and 2013. To this end, we sequenced complete nsp12, $\mathrm{S}$, and $\mathrm{N}$ genes of 15 molecular isolates of HCoV-OC43 from clinical samples and compared them to available data from the USA, Belgium, and Hong-Kong. A new cluster $\mathrm{E}$ was invariably detected from nsp12, S, and $\mathrm{N}$ data while the analysis of nsp12 and $\mathrm{N}$ genes revealed the existence of new $\mathrm{F}$ and $\mathrm{G}$ clusters respectively. The association of these different clusters of genes in our specimens led to the description of thirteen genetically distinct genotypes, among which eight recombinant viruses were discovered. Identification of these recombinant viruses, together with temporal analysis
\end{abstract}


and tMRCA estimation, provides important information for understanding the dynamics of the evolution of these epidemic coronaviruses.

Keywords: genotype; sequencing; coronavirus; phylogeny; recombination; HCoV-OC43

\section{Introduction}

Coronaviruses belong to the Coronaviridae family in Nidovirales order [1]. This order comprises the largest enveloped single-strand RNA viruses, and includes three other families; Arterividae, Roniviridae, and the most recently described Mesoniviridae [2]. Coronaviruses are divided into four genera named Alpha-, Beta-, Delta-, and Gammacoronavirus, based on the phylogenetic distance of highly conserved domains [3-5]. Alphacoronaviruses are divided into two subgroups or clades, A and B or 1 and 2, and betacoronaviruses are divided into four subgroups or clades, A to D or 1 to 4 [6,7].

Coronaviruses infect a wide range of avian or mammalian species, and are responsible for enteric or respiratory infections [8-10]. Six human coronaviruses (HCoVs) have been identified of which four are in circulation: HCoV-229E, HCoV-NL63 (genus Alphacoronavirus), and HCoV-OC43 and HCoV-HKU1 (genus Betacoronavirus clade A). These four viruses are responsible for mild upper-respiratory tract infections, yet cause more severe respiratory pathologies in infants, immunocompromised patients and elderly people [11-22]. The other two human coronaviruses, Severe Acute Respiratory Syndrome coronavirus (SARS-CoV) and Middle-East Respiratory Syndrome coronavirus (MERS-CoV), belonging to Betacoronavirus genus, clades $\mathrm{B}$ and $\mathrm{C}$ respectively, cause severe respiratory pathologies. SARS-CoV circulated in 2002-2003 and caused a global outbreak with more than 8000 cases, and produced a rate of fatal cases of nearly 10\%; and MERS-CoV emerged in 2012 in the Middle-East, causing severe pneumonia similar to that from SARS [23-25]. To date, 961 laboratory confirmed cases of MERS-CoV infection, including 418 fatal cases, have been reported [26].

The first isolation of $\mathrm{HCoV}-\mathrm{OC} 43$ from a nasopharyngeal wash was reported in a publication in 1967 [13]. In 2004 and 2005 respectively, St-Jean et al. and Vijgen et al. published the first complete genome sequence of HCoV-OC43 [27,28]. The genome of HCoV-OC43 consists of a positive sense, single-stranded RNA molecule, which is 30,738 bases in length, encoding 11 ORFs. The first two ORFs (1a and 1b) starting from the $5^{\prime}$ end of the molecule account for approximately two-thirds of the genome. The last third towards the $3^{\prime}$ end containing genes coding the hemagglutinin esterase (HE), the spike glycoprotein $(\mathrm{S})$, the envelop protein (E), the matrix glycoprotein (M), and the nucleocapsid phosphoprotein $(\mathrm{N})$. The mechanism of replication of $\mathrm{HCoV}-\mathrm{OC} 43$ uses a low fidelity RNA dependent RNA polymerase (RdRp) characterized by a mutation rate of $10^{-3}$ to $10^{-4}$ mutation/nucleotide/round of replication [29,30]. The replication of coronavirus genomes requires a step during which a set of subgenomic RNAs is generated. This mechanism contributes to promoting homologous recombination events [31]. Due to its genomic plasticity, coronaviruses are characterized by a high potential of evolution, adaptation, and interspecies jumping [7]. Coronaviruses are also characterized by their interspecies and intraspecies variability. This study is focused on the latter.

In 2006, Vabret et al. observed the co-circulation of genetic variants inside the species HCoV-OC43 by analysing the partial $\mathrm{S}$ gene (nt 23,449 to 26,332, reference to HCoV-OC43 ATCC-VR759 
AY391777), coding for the glomerular part of the S protein (sub-unit S1) of $7 \mathrm{HCoV}-\mathrm{OC} 43$ s from clinical samples collected in 2003 at the University Hospital of Caen. They observed four genetically distinct subgroups. One of the subgroups constitutes an outlier group located between HCoV-OC43 and Bovine coronavirus (BCoV), containing a 12-nucleotide deletion in common with $\mathrm{BCoV}$ but not with other $\mathrm{HCoV}-\mathrm{OC} 43 \mathrm{~s}$ [32]. More recently, four genetically distinct $\mathrm{HCoV}-\mathrm{OC} 43$ genotypes have been identified from respiratory specimens sampled at the Public Hospital of China over a 7-year period (2004 to 2011) [33]. In this study, Lau et al. based their genotype definition upon the complete sequence of nsp12, S and $\mathrm{N}$ genes [33]. Genotype A contains the prototype VR759 strain isolated in 1967. Genotypes B and C are two naturally circulating HCoV-OC43s. Genotype D is a recombinant virus of genotypes B and C, obtained from a specimen from 2004 [33]. Based on a bootscan analysis of the complete genome of the $3 \mathrm{HCoV}-\mathrm{OC} 43$ s belonging to the circulating genotypes $\mathrm{B}, \mathrm{C}$, and $\mathrm{D}$, it was assumed that a hot spot was likely located between the nsp12 and S genes, more precisely at the nsp12/nsp13 junction. Our objective was to investigate the presence, the temporal distribution and the recombination patterns of $\mathrm{HCoV}-\mathrm{OC} 43$ in Lower Normandy over 13 years (2001 to 2013), using the methodology and the HCoV-OC43 genotype definition elaborated by Lau et al. in 2011 [33]. This study focuses on the sequences of the nsp12, S, and $\mathrm{N}$ genes of $15 \mathrm{HCoV}-\mathrm{OC} 43 \mathrm{~s}$ detected in respiratory specimens sampled from 2001 to 2013.

\section{Results}

\subsection{Sequencing of nsp 12, $S$ and $N$ Genes}

Of the $15 \mathrm{HCoV-OC43s}$ and the prototype strain VR759, six, eight, and three overlapping sequences were obtained for the nsp12, S, and $\mathrm{N}$ genes, respectively. After assembly, these overlapping fragments encompassed the full nsp12, S, and N genes. In this study, all HCoV-OC43s including the VR759 prototype strain are associated with three accession numbers in GenBank, for nsp12, S, and N genes (Table 1). The sequences of HCoV-OC43s were named according to the following nomenclature: Virus/FRA-EPI/location of sampling/year of sampling/specimen number. EPI is an abbreviation for EPICOREM consortium. For the prototype strain, the specimen number is replaced by VR759.

Table 1. GenBank accession numbers associated with the sequences used in this study.

\begin{tabular}{cccc}
\hline \multirow{2}{*}{ Name of Sequences } & \multicolumn{3}{c}{ Accession Number } \\
\cline { 2 - 4 } & nsp12 & S & N \\
\hline HCoV-OC43/FRA_EPI/Caen/1967/VR759 & KF963213 & KF963229 & KF963245 \\
HCoV-OC43/FRA_EPI/Caen/2001/01 & KF963214 & KF963230 & KF963246 \\
HCoV-OC43/FRA_EPI/Caen/2001/02 & KF963215 & KF963231 & KF963247 \\
HCoV-OC43/FRA_EPI/Caen/2002/03 & KF963216 & KF963232 & KF963248 \\
HCoV-OC43/FRA_EPI/Caen/2002/04 & KF963217 & KF963233 & KF963249 \\
HCoV-OC43/FRA_EPI/Caen/2003/05 & KF963218 & KF963234 & KF963250 \\
HCoV-OC43/FRA_EPI/Caen/2004/06 & KF963219 & KF963235 & KF963251 \\
HCoV-OC43/FRA_EPI/Caen/2005/07 & KF963220 & KF963236 & KF963252 \\
HCoV-OC43/FRA_EPI/Caen/2006/08 & KF963221 & KF963237 & KF963253 \\
HCoV-OC43/FRA_EPI/Caen/2007/09 & KF963222 & KF963238 & KF963254 \\
HCoV-OC43/FRA_EPI/Caen/2008/10 & KF963223 & KF963239 & KF963255 \\
\hline
\end{tabular}


Table 1. Cont.

\begin{tabular}{|c|c|c|c|}
\hline \multirow{2}{*}{ Name of Sequences } & \multicolumn{3}{|c|}{ Accession Number } \\
\hline & nsp12 & $\mathbf{S}$ & $\mathbf{N}$ \\
\hline HCoV-OC43/FRA_EPI/Caen/2009/11 & KF963224 & KF963240 & KF963256 \\
\hline HCoV-OC43/FRA_EPI/Caen/2010/12 & KF963225 & KF963241 & KF963257 \\
\hline HCoV-OC43/FRA_EPI/Caen/2011/13 & KF963226 & KF963242 & KF963258 \\
\hline HCoV-OC43/FRA_EPI/Caen/2012/14 & KF963227 & KF963243 & KF963259 \\
\hline \multirow[t]{2}{*}{ HCoV-OC43/FRA_EPI/Caen/2013/15 } & KF963228 & KF963244 & KF963260 \\
\hline & \multicolumn{3}{|c|}{ full genome } \\
\hline OC43/human/USA/971-5/1997 & \multicolumn{3}{|c|}{ KF530099 } \\
\hline OC43/human/USA/965-6/1996 & \multicolumn{3}{|c|}{ KF530098 } \\
\hline OC43/human/USA/832-27/1983 & \multicolumn{3}{|c|}{ KF530093 } \\
\hline OC43/human/USA/008-5/2000 & \multicolumn{3}{|c|}{ KF530092 } \\
\hline OC43/human/USA/911-58/1991 & \multicolumn{3}{|c|}{ KF530091 } \\
\hline OC43/human/USA/931-85/1993 & \multicolumn{3}{|c|}{ KF530090 } \\
\hline OC43/human/USA/901-54/1990 & \multicolumn{3}{|c|}{ KF530088 } \\
\hline OC43/human/USA/872-5/1987 & \multicolumn{3}{|c|}{ KF530086 } \\
\hline OC43/human/USA/951-18/1995 & \multicolumn{3}{|c|}{ KF530084 } \\
\hline OC43/human/USA/8912-37/1989 & \multicolumn{3}{|c|}{ KF530073 } \\
\hline OC43/human/USA/925-1/1992 & \multicolumn{3}{|c|}{ KF530071 } \\
\hline OC43/human/USA/007-11/2000 & \multicolumn{3}{|c|}{ KF530068 } \\
\hline OC43/human/USA/953-23/1995 & \multicolumn{3}{|c|}{ KF530062 } \\
\hline HK04_01 & \multicolumn{3}{|c|}{ JN129834 } \\
\hline HK04_02 & \multicolumn{3}{|c|}{ JN129835 } \\
\hline 19572 Belgium 2004 & \multicolumn{3}{|c|}{ AY903460 } \\
\hline 87309 Belgium 2003 & \multicolumn{3}{|c|}{ AY903459 } \\
\hline HCoV-OC43 VR759 [28] & \multicolumn{3}{|c|}{ NC_005147 } \\
\hline HCoV-OC43 VR759 [34] & \multicolumn{3}{|c|}{ AY391777 } \\
\hline BCoV Mebus & \multicolumn{3}{|c|}{ U00735 } \\
\hline BCoV Kakewaga & \multicolumn{3}{|c|}{ AB354579 } \\
\hline BCoV Quebec & \multicolumn{3}{|c|}{ AF220295 } \\
\hline
\end{tabular}

\subsection{Phylogenetic Analysis of nsp12, S, and N Genes}

The phylogenetic analysis was conducted by comparing the topology of the three phylogenetic trees obtained from the nsp12, S, and N genes. Figure 1 shows the three trees obtained by the neighbor joining method [35]. On each tree, five to six clusters were observed, including an outlier group compounded with the three BCoVs sequences Mebus, Kakegawa, and Quebec that root HCoV-OC43 sequences [36-38]. The clustering is supported with high bootstrap values. We used part of the nomenclature of genotypes proposed by Lau et al. in 2011 [33]. These authors described the genotypes $\mathrm{A}, \mathrm{B}, \mathrm{C}$, and D from nsp12, S and $\mathrm{N}$ genes. We used this nomenclature to name each genotype using three letters corresponding to clusters in which the different sequences are located in nsp12, $\mathrm{S}$, and $\mathrm{N}$ trees, respectively. Following this nomenclature, genotype $\mathrm{D}$ is a recombinant genotype $\mathrm{B} / \mathrm{C} / \mathrm{C}$. The three previously described clusters - A, B, and C-are observed in our three gene trees in addition to a newly described cluster $\mathrm{E}$. The nsp12 tree depicts a new cluster we named " $\mathrm{F}$ " that roots all other 
$\mathrm{HCoV}-\mathrm{OC} 43 \mathrm{~s}$. According to the $\mathrm{N}$ gene, a sixth cluster we named "G" separates from cluster $\mathrm{E}$ and roots clusters $\mathrm{A}, \mathrm{B}$, and $\mathrm{C}$. Among the set of sequences obtained from the $16 \mathrm{HCoV}-\mathrm{OC} 43 \mathrm{~s}$ of our study, three are distributed in two non-recombinant genotypes as follows: the VR759 sequence belongs to the genotype AAA; HCoV-OC43/FRA-EPI/Caen/2003/05 and HCoV-OC43/FRA-EPI/Caen/2006/08 belong to genotype BBB. The 13 other sets of sequences are allocated among six recombinant genotypes as follows: five genotypes BCC, defined as genotype D by Lau et al.; two genotypes CEE, three genotypes $\mathrm{CBE}$, one genotype $\mathrm{BCG}$, one genotype $\mathrm{CCE}$, and one genotype CEB. Among the 14 American sets of sequences, seven are distributed into two non-recombinant genotypes as follows: one CCC and six EEE. The seven other sets of sequences are distributed among four recombinant genotypes as follows: three genotypes CCA, two genotypes $\mathrm{FCB}$, one genotype $\mathrm{CCB}$, and one genotype CEE. Among the two sets of sequences from Hong-Kong, one is a non-recombinant genotype CCC and the other is a recombinant genotype BCC. Among the two HCoV-OC43s from Belgium, one is a non-recombinant genotype BBB and the other is a recombinant genotype BCC. Finally, the two last sequences of HCoV-OC43 VR759 (accession number: AY391777 and NC005147) belong to the non-recombinant genotype AAA [28,39]. The results of the phylogenetic analysis are summarized in Table 2 .

\subsection{Insights in the Evolutionary History}

The two alignments constructed from the 39 dated sequences of $\mathrm{S}$ and $\mathrm{N}$ genes have been used to set up a molecular clock calibration in order to estimate the date of emergence of mean clusters. Figure 2 shows Bayesian trees inferred from the alignments of $\mathrm{S}$ and $\mathrm{N}$ genes. The dates of emergence of the clusters and the corresponding 95\% Highest Posterior Density (95\% HPD) are indicated. Based on the sequence data of the $\mathrm{S}$ gene, the tMRCA of $\mathrm{BCoV}$ and $\mathrm{HCoV}-\mathrm{OC} 43$ is estimated in 1885, with a 95\% HPD from 1858 to 1907 . For the $\mathrm{HCoV}-\mathrm{OC} 43$, cluster $\mathrm{E}$ is predicted to have emerged in 1943 (95\% HPD 1933 to 1952), and genotype A is estimated to have emerged in 1951 (95\% HPD, 1943 to 1959). Genotypes B and C are predicted to have emerged from their tMRCA in 1982 (95\% HPD, 1980 to 1983). The molecular clock conducted from sequence data of $\mathrm{N}$ gene allows us to estimate the tRMCA of BCoV and HCoV-OC43 in 1879 (95\% HPD, 1822 to 1923). Genotypes E and A are predicted to have emerged in 1932 (95\% HPD, 1904 to 1952) and 1957 (95\% HPD, 1950 to 1972), respectively. Genotypes B and C are predicted to have emerged from their tMRCA in 1984 (95\% HPD, 1976 to 1991). 

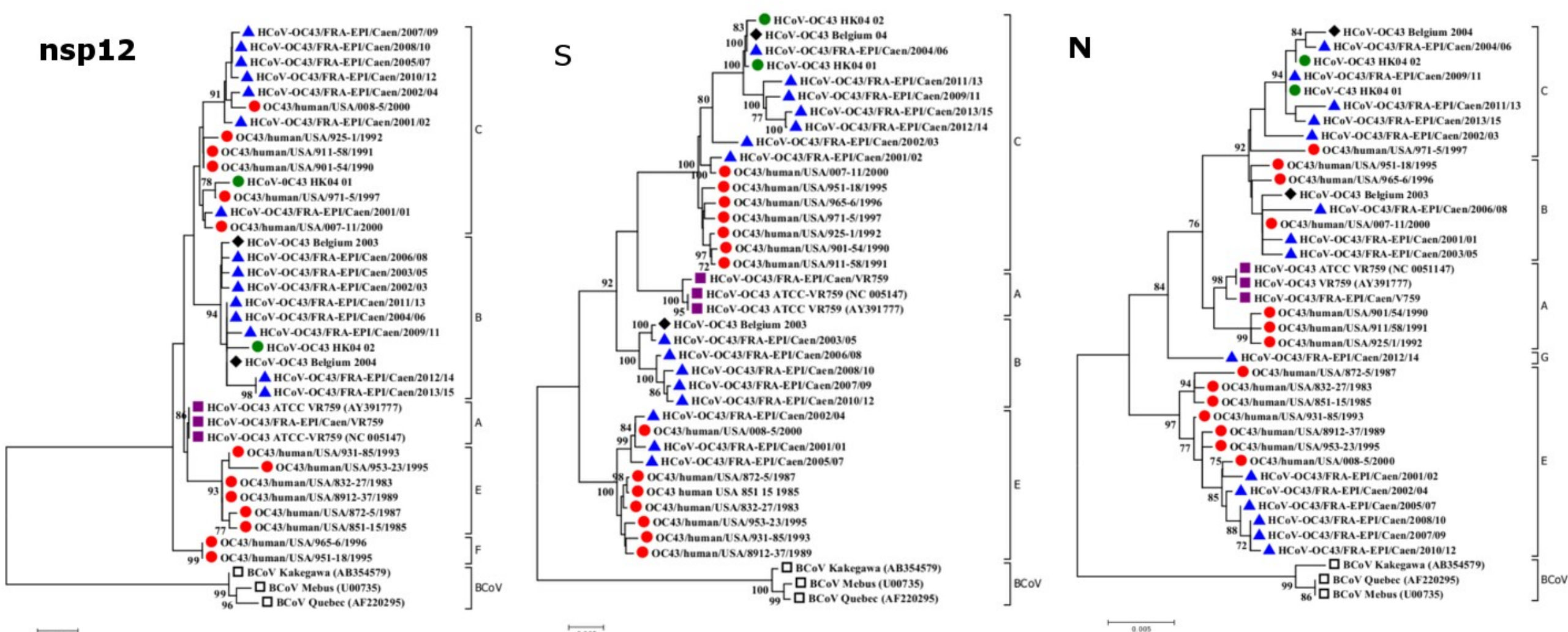

Figure 1. Phylogenetic analysis of the complete nsp12, S, and N genes of the $36 \mathrm{HCoV}-\mathrm{OC} 43$ strains and 3 BCoV strains. The phylogenetic trees were constructed by the neighbor joining method [35]. Bootstrap values were calculated from 1000 replicates. Bootstrap values over $70 \%$ are shown [40]. The evolutionary distances were computed using the Kimura 2-parameter method (kimura) and units are the number of base substitutions per site. Evolutionary analyses were conducted in MEGA, version 6.06. [41]. Blue triangle, isolates from Caen; red circle, isolates from USA; green circle, isolates from Hong-Kong; black diamond, isolates from Belgium; purple square, ATCC-VR759 strains, empty black square, $\mathrm{BCoV}$ strains. 

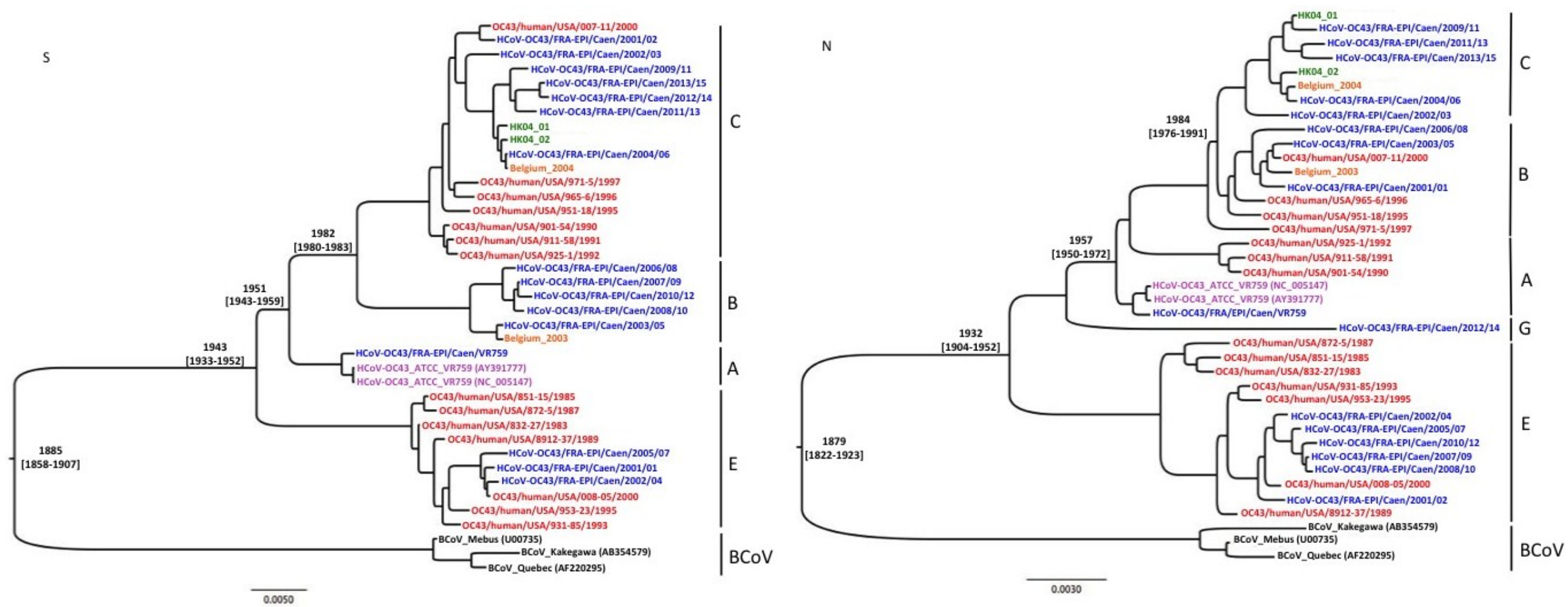

Figure 2. Results of Bayesian analysis based on $\mathrm{S}$ and $\mathrm{N}$ gene sequence data. Inferences were calculated with the one parametric coalescent model with a constant size, under the TN93+G substitution parameter, using the BEAST package (version 1.8.2) [42]. The length of MCMC was fixed at $10^{8}$ states for $\mathrm{S}$ and $\mathrm{N}$ genes. The dates of emergence of mean clusters are indicated, associated with the 95\% HPD. 
Table 2. Results of phylogenetic analysis of complete nsp12, S and N genes. * Genotype D, according to the definition from Lau et al. [33].

\begin{tabular}{|c|c|c|c|c|}
\hline Sequences & nsp12 & $\mathbf{S}$ & $\mathbf{N}$ & Genotype \\
\hline \multicolumn{5}{|l|}{ France } \\
\hline HCoV-OC43/FRA_EPI/Caen/2001/01 & $\mathrm{C}$ & $\mathrm{E}$ & $\mathrm{B}$ & CEB \\
\hline HCoV-OC43/FRA_EPI/Caen/2001/02 & $\mathrm{C}$ & $\mathrm{C}$ & $\mathrm{E}$ & $\mathrm{CCE}$ \\
\hline HCoV-OC43/FRA_EPI/Caen/2002/03 & B & $\mathrm{C}$ & $\mathrm{C}$ & $\mathrm{BCC} *$ \\
\hline HCoV-OC43/FRA_EPI/Caen/2002/04 & $\mathrm{C}$ & $\mathrm{E}$ & $\mathrm{E}$ & CEE \\
\hline HCoV-OC43/FRA_EPI/Caen/2003/05 & B & $\mathrm{B}$ & $\mathrm{B}$ & BBB \\
\hline HCoV-OC43/FRA_EPI/Caen/2004/06 & B & $\mathrm{C}$ & $\mathrm{C}$ & $\mathrm{BCC} *$ \\
\hline HCoV-OC43/FRA_EPI/Caen/2005/07 & $\mathrm{C}$ & $\mathrm{E}$ & $\mathrm{E}$ & CEE \\
\hline HCoV-OC43/FRA_EPI/Caen/2006/08 & B & $\mathrm{B}$ & $\mathrm{B}$ & BBB \\
\hline HCoV-OC43/FRA_EPI/Caen/2007/09 & $\mathrm{C}$ & $\mathrm{B}$ & $\mathrm{E}$ & $\mathrm{CBE}$ \\
\hline HCoV-OC43/FRA_EPI/Caen/2008/10 & $\mathrm{C}$ & $\mathrm{B}$ & $\mathrm{E}$ & $\mathrm{CBE}$ \\
\hline HCoV-OC43/FRA_EPI/Caen/2009/11 & B & $\mathrm{C}$ & $\mathrm{C}$ & $\mathrm{BCC} *$ \\
\hline HCoV-OC43/FRA_EPI/Caen/2010/12 & $\mathrm{C}$ & $\mathrm{B}$ & $\mathrm{E}$ & $\mathrm{CBE}$ \\
\hline HCoV-OC43/FRA_EPI/Caen/2011/13 & B & $\mathrm{C}$ & $\mathrm{C}$ & $\mathrm{BCC} *$ \\
\hline HCoV-OC43/FRA_EPI/Caen/2012/14 & B & $\mathrm{C}$ & G & BCG \\
\hline HCoV-OC43/FRA_EPI/Caen/2013/15 & $\mathrm{B}$ & $\mathrm{C}$ & $\mathrm{C}$ & $\mathrm{BCC} *$ \\
\hline \multicolumn{5}{|l|}{ USA } \\
\hline OC43/human/USA/832-27/1983 & $\mathrm{E}$ & $\mathrm{E}$ & $\mathrm{E}$ & EEE \\
\hline OC43/human/USA/851-15/1985 & $\mathrm{E}$ & $\mathrm{E}$ & $\mathrm{E}$ & EEE \\
\hline OC43/human/USA/872-5/1987 & $\mathrm{E}$ & $\mathrm{E}$ & $\mathrm{E}$ & EEE \\
\hline OC43/human/USA/8912-37/1989 & $\mathrm{E}$ & $\mathrm{E}$ & $\mathrm{E}$ & EEE \\
\hline OC43/human/USA/901-54/1990 & $\mathrm{C}$ & $\mathrm{C}$ & A & CCA \\
\hline OC43/human/USA/911-58/1991 & $\mathrm{C}$ & $\mathrm{C}$ & A & CCA \\
\hline OC43/human/USA/925-1/1992 & $\mathrm{C}$ & $\mathrm{C}$ & A & CCA \\
\hline OC43/human/USA/931-85/1993 & $\mathrm{E}$ & $\mathrm{E}$ & $\mathrm{E}$ & EEE \\
\hline OC43/human/USA/953-23/1995 & $\mathrm{E}$ & $\mathrm{E}$ & $\mathrm{E}$ & EEE \\
\hline OC43/human/USA/951-18/1995 & $\mathrm{F}$ & $\mathrm{C}$ & $\mathrm{B}$ & FCB \\
\hline OC43/human/USA/965-6/1996 & $\mathrm{F}$ & $\mathrm{C}$ & $\mathrm{B}$ & FCB \\
\hline OC43/human/USA/971-5/1997 & $\mathrm{C}$ & $\mathrm{C}$ & $\mathrm{C}$ & $\mathrm{CCC}$ \\
\hline OC43/human/USA/007-11/2000 & $\mathrm{C}$ & $\mathrm{C}$ & $\mathrm{B}$ & $\mathrm{CCB}$ \\
\hline OC43/human/USA/008-5/2000 & $\mathrm{C}$ & $\mathrm{E}$ & $\mathrm{E}$ & CEE \\
\hline \multicolumn{5}{|l|}{ Hong-Kong } \\
\hline HK04_01 & $\mathrm{C}$ & $\mathrm{C}$ & $\mathrm{C}$ & $\mathrm{CCC}$ \\
\hline HK04_02 & $\mathrm{B}$ & $\mathrm{C}$ & $\mathrm{C}$ & $\mathrm{BCC} *$ \\
\hline \multicolumn{5}{|l|}{ Belgium } \\
\hline BE03 & B & $\mathrm{B}$ & $\mathrm{B}$ & BBB \\
\hline $\mathrm{BEO} 4$ & B & $\mathrm{C}$ & $\mathrm{C}$ & $\mathrm{BCC} *$ \\
\hline VR759 & & & & \\
\hline HCoV-OC43 (AY391777) & A & A & A & AAA \\
\hline HCoV-OC43 VR759 (NC005147) & A & A & A & AAA \\
\hline HCoV-OC43/FRA_EPI/Caen/1967/VR759 & $\mathrm{A}$ & A & A & $\mathrm{AAA}$ \\
\hline
\end{tabular}




\section{Discussion}

The $4 \mathrm{HCoVs}$ - OC43, 229E, NL63, and HKU1-belong to the viral molecular panel tested during the virological routine diagnostics of acute respiratory infections in humans. Among these four circulating HCoVs, OC43 and NL63 seem to show the greatest prevalence and incidence. These viruses also proved to be the viruses encountered at the earliest age of childhood [43]. These HCoVs circulate widely in epidemic form in the general population, causing infections that are most often benign. Infants, immunosuppressed patients, and very elderly patients may however develop very severe pathologies. These four circulating HCoVs must be distinguished from the emerging HCoVs, SARS-CoV, and MERS-CoV, causes of much graver and potentially fatal respiratory pathologies. Control of the latter HCoVs requires the implementation of international health measures [44-46].

The evolutionary potential of coronaviruses brings into play point mutations and recombination events. Such genetic diversity generated in this way may have an impact on the performance of molecular detection techniques used in the virological diagnostic process. The study of intra-specific diversity is thus useful in the monitoring of means of detection. Specifically, it allows for the detection of new circulating variants, and provides information about the evolutionary dynamics of the family of respiratory viruses being monitored, with special attention given to coronaviruses. Our study focuses solely on the HCoV-OC43.

The first analyses of the $\mathrm{HCoV}-\mathrm{OC} 43 \mathrm{~S}$ gene were conducted in 2005 and revealed a potential spatial and temporal distribution of genetic clusters [34]. In 2011, S. Lau and his colleagues were the first to propose a genotypic classification of HCoV-OC43 from the complete sequencing of three genes: Nsp12 (RdRp), S (spike), and N (nucleocapsid). Four genotypes-A, B, C, and D-were identified: genotype A corresponds to the sequences of the HCoV-OC43 prototype strain VR759, adapted to culture cell line HRT-18 (human adenocarsinoma rectal) or RD (rhabdomyosarcoma); genotypes $\mathrm{B}$ and $\mathrm{C}$ correspond to contemporary circulating strains, and genotype $\mathrm{D}$ is described as a recombinant $\mathrm{B} / \mathrm{C}$ virus. This study was performed on $29 \mathrm{HCoV}-\mathrm{OC} 43 \mathrm{~s}$ detected in respiratory samples collected in Hong Kong over a period of 7 years (2004 to 2011), in a non-homogenous temporal distribution. More precisely, the majority of these samples (18 of 29) were collected in 2004, and the others (11 of 29) fall between 2005 and 2011 [33]. Our study defines the genotype of 15 HCoV-OC43s detected from upper respiratory samples frozen at $-80{ }^{\circ} \mathrm{C}$, collected over a period of 13 years from 2001 to 2013 at the rate of one to two samples per year. The $15 \mathrm{HCoV}-\mathrm{OC} 43 \mathrm{~s}$ were detected in patients hospitalized in our university hospital. Of these 15 patients, 10 were male and five were female, at ages from 4 months to 67 years. The symptomatology proved variable (Table 3). The infections were essentially located in upper and lower respiratory airways. The presence of associated gastrointestinal symptoms in some patients should be noted. The prototype strain HCoV-OC43 ATCC VR759, grown on cell line HRT-18, was used as a control, allowing for the validation of the methodology used by Lau et al. [33]. This methodology had to be adapted since some of the primers published by the authors did not allow for amplification of all the HCoV-OC43s. 
Table 3. Clinical features of the 15 french patients. ${ }^{a}$ Mo., month; yr., year; d., days. ${ }^{b} \mathrm{M}$, male; F, female. ${ }^{\mathrm{c}} \mathrm{Na}$, not available.

\begin{tabular}{|c|c|c|c|c|c|}
\hline Specimen & $\begin{array}{l}\text { Age at Time } \\
\text { of Sampling a }\end{array}$ & $\begin{array}{c}\text { Date If Sampling } \\
\text { (Day/Mo/Year) }\end{array}$ & Gender b & $\begin{array}{c}\text { Duration of } \\
\text { Hospitalization }{ }^{c}\end{array}$ & Final Diagnosis \\
\hline Caen/2001/01 & 3 mo. & $02 / 20 / 2001$ & M & na & na \\
\hline Caen/2001/02 & $3 \mathrm{yr}$. & $02 / 07 / 2011$ & M & $4 \mathrm{~d}$. & gastroenteritis \\
\hline Caen/2002/03 & $5 \mathrm{mo}$. & $03 / 12 / 2002$ & M & $15 \mathrm{~d}$. & $\begin{array}{c}\text { nasoparyngitis, seromucous } \\
\text { bilateral otitis }\end{array}$ \\
\hline Caen/2002/04 & 4 mo. & $02 / 21 / 2002$ & M & na & na \\
\hline Caen/2003/05 & $1 \mathrm{yr}$. & $03 / 17 / 2003$ & M & na & na \\
\hline Caen/2004/06 & $1 \mathrm{yr}$. & $02 / 20 / 2004$ & $\mathrm{~F}$ & $4 \mathrm{~d}$. & gastroenteritis \\
\hline Caen/2005/07 & $2 \mathrm{yr}$. & $02 / 07 / 2005$ & $\mathrm{~F}$ & $4 \mathrm{~d}$. & gastroenteritis, acute otitis media \\
\hline Caen/2006/08 & $11 \mathrm{mo}$. & $04 / 12 / 2006$ & M & na & na \\
\hline Caen/2007/09 & $6 \mathrm{mo.}$ & $06 / 16 / 2007$ & M & na & na \\
\hline Caen/2008/10 & $3 \mathrm{yr}$. & $01 / 17 / 2008$ & M & $5 \mathrm{~d}$. & acute etmoidis \\
\hline Caen/2009/11 & $3 \mathrm{mo}$ & $11 / 02 / 2009$ & M & $3 \mathrm{~d}$. & bronchitis \\
\hline Caen/2010/12 & $1 \mathrm{yr}$. & $12 / 17 / 2010$ & M & na & bronchitis \\
\hline Caen/2011/13 & 4 mo. & $11 / 20 / 2010$ & M & na & na \\
\hline Caen/2012/14 & $67 \mathrm{yr}$. & $03 / 30 / 2012$ & M & na & confusion \\
\hline Caen/2013/15 & 53 yr. & $03 / 18 / 2013$ & $\mathrm{~F}$ & na & Upper respiratory infection \\
\hline
\end{tabular}

The alignments of complete sequences of three genes - nsp12, S, and N-of the $15 \mathrm{HCoV}-\mathrm{OC} 43 \mathrm{~s}$ used in this study were generated and enriched by the addition of the corresponding sequences of several complete HCoV-OC43 genomes available in GenBank: two prototype sequences of HCoV-OC43s VR759 published by Vijgen et al. in 2005 [28]; two HCoV-OC43s from 2004 reported by Lau et al. in 2011 (HK04_01, HK04_02) [33]; and two HCoV-OC43s described in Belgium in 2003 and 2004 (Belgium 03 and Belgium 04 respectively) [34]. This data set was completed at the end of 2013 with the publication of 41 complete HCoV-OC43 sequences by Town et al. in GenBank. These sequences correspond to $41 \mathrm{HCoV}-\mathrm{OC} 43 \mathrm{~s}$ identified in the USA (Maryland) between 1983 and 2000. Of these 41 sequences, we selected 14 that reflect the genetic diversity and temporal distribution of the whole, and we introduced them into the alignment. In the end, three respective alignments of complete genes - nsp12, S, and $\mathrm{N}$ - of $36 \mathrm{HCoV}-\mathrm{OC} 43 \mathrm{~s}$ identified in three different regions of the world (Hong Kong, Europe, and the USA) over a period of 30 years (from 1983 to 2013) were generated and analyzed. They served as the basis for the construction of phylogenetic trees. Within the three phylogenetic trees-nsp12, S, and $\mathrm{N}$-we identified six different clusters named A, B, C, E, F, and G. A comparative study of the topology of phylogenetic trees corresponding to several genes of the same virus is often used in phylogeny to define recombinant viruses $[33,47,48]$. We thus compared the topology of nsp12, S, and $\mathrm{N}$ trees by observing the contextual position of the corresponding sequences of the same HCoV-OC43 molecular isolate. The complexity of the results of our analysis led us to establish a nomenclature allowing for the simplest expression of the genotypes: each genotype is expressed in the form of a three-letter code, linking each one to the member cluster of the different nsp12, S, and N sequences. From here, we defined 13 different genotypes, of which four were non-recombinant (AAA, BBB, CCC, and EEE). The nine remaining genotypes correspond to different 
recombinant genotypes (Table 2). Among the $36 \mathrm{HCoV}-\mathrm{OC} 43$ sequences examined in this study, $14 \mathrm{HCoV}-\mathrm{OC} 43 \mathrm{~s}$ of so-called non-recombinant genotypes were found: three HCoV-OC43s of genotype AAA corresponding to prototype strains VR759; three HCoV-OC43s of genotype BBB (origin, France 2003 and 2006, and Belgium 2003); two HCoV-OC43s of genotype CCC (origin, USA 1997 and Hong Kong 2004); and lastly, six HCoV-OC43s of genotype EEE, all originating in the USA, and having circulated from 1983 to 1995 . The foremost important point to underline is that these results suggest a high level of recombination among circulating HCoV-OC43 epidemic strains. The limited number of sequences studied in time and space does not allow for conclusive evidence of a possible temporal and spatial distribution of different genotypes HCoV-OC43. However, it should be noted that the $\mathrm{E}$ cluster has not yet been described. In the present study, it is identified only in HCoV-OC43 sequences from the USA and France and data suggest that it has been circulating since the 1940s after being the first known group to differentiate from $\mathrm{BCoV}$.

The analysis of alignments shows that cluster $\mathrm{E}$ is characterized by a deletion of 12 nucleotides in the $\mathrm{S}$ gene, more precisely in the $\mathrm{S} 1$ gene that codes the glomerular part of the $\mathrm{S}$ protein (position 24,091-24,102, GenBank accession number NC_005147). This deletion of 12 nucleotides in the coding phase results in a deletion of 4 amino acids, TQDG, in the corresponding protein sequence. This deletion was also present in the bovine coronavirus strains used in the alignment, as shown in Figure 3. The analysis of $105 \mathrm{~S} 1 \mathrm{BCoV}$ sequences available in GenBank shows that this deletion is expressed in all BCoVs, and described in bovine coronaviruses associated with both enteric and respiratory tropisms. This deletion is therefore not a specific marker of viral tropism. It is located in the lectin domain of the $\mathrm{S} 1$ protein of $\mathrm{BCoV}$, and is involved in the attachment of the virus to the cellular receptor, identified as a derivative of neuraminic acid (N-acetyl-9-O-acetylneuraminic acid or Neu5,9Ac2). No protein receptor has yet been described for the $\mathrm{BCoV}$ or, more broadly, for Betacoronavirus bovine-like clade A. We analyzed all the sequences of this $\mathrm{S} 1 \mathrm{HCoV}-\mathrm{OC} 43$ region available in GenBank, for a total of 108 sequences, and the deletion of 12 nucleotides is found in only 27 of them. Of these 27 sequences, 19 correspond to HCoV-OC43s detected in respiratory samples from the USA and sequenced by Town et al. Four correspond to HCoV-OC43s detected in France (three in the current study, and one published by our team in 2006); two correspond to HCoV-OC43 sequences deposited by Browlin et al. under patent in the United Kingdom (EP2182066 and WO2004011651), and finally, the remaining two correspond to HCoV-OC43s adapted to Vero cells and MDCK I, published in 1996 by F. Künkel and Herrler G [49]. The HCoV-OC43 E cluster is phylogenetically close to the $\mathrm{BCoV}$ cluster, and the presence of the genetic marker common to these two clusters provides an argument supporting the hypothesis put forth by Vijgen in 2006 that would identify a crossing of the species barrier occurring from cattle to human [39]. A molecular clock analysis was inferred using the BEAST software version 1.8.1 (http://beast.bio.ed.ac.uk) based on sequence alignments of $\mathrm{S}$ and $\mathrm{N}$ genes. Sequences of the nsp12 gene were not used because they were uninformative due to the low variability of this gene. Results were compared with those previously obtained by Vijgen from 20 Betacoronavirus clade A sequences (nine BCoVs, three PHEVs, and eight $\mathrm{HCoV-OC43s).} \mathrm{It} \mathrm{should} \mathrm{be} \mathrm{noted} \mathrm{that} \mathrm{eight} \mathrm{sets} \mathrm{of} \mathrm{HCoV-OC43} \mathrm{sequences} \mathrm{used} \mathrm{in} \mathrm{the} \mathrm{analysis} \mathrm{of}$ Vijgen et al. in 2006 were obtained from samples collected over a very short period between 2003 and 2004 [39]. 


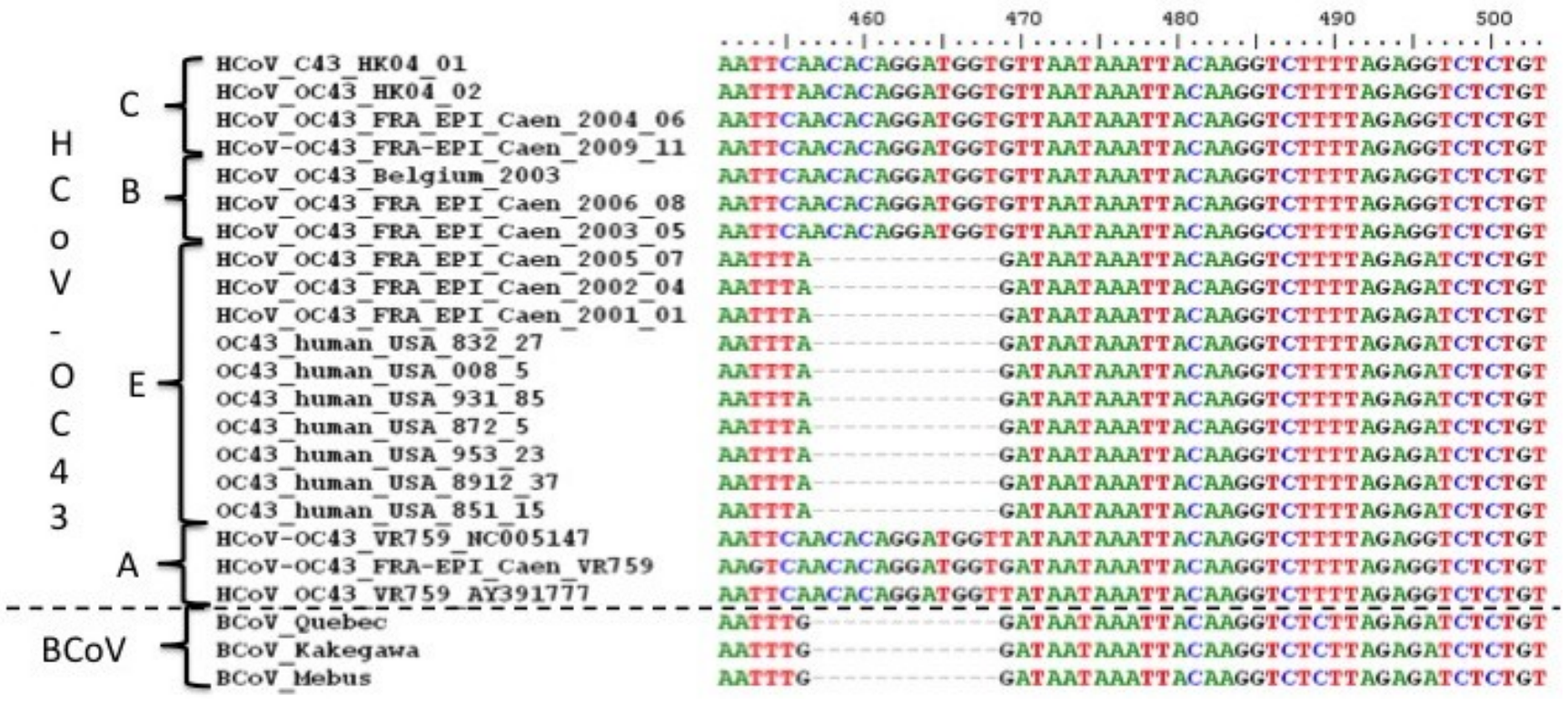

Figure 3. Alignement of some of the $\mathrm{S}$ genes sequences. This aligment was made using Bioedit Software version 7.2.5 (Ibis Biosciences, Carlsbad, CA, USA).

In 2013, Wertheim et al. demonstrated that a strong purifying selection could lead to the underestimation of the evolutionary rate of coronaviruses. In this study, the authors proposed an alternative theory of origin for all coronaviruses. They estimate the tMRCA of coronaviruses infecting mammalian species (Alpha- and Betacoronavirus genus) and avian species (Gamma- and Deltacoronavirus genus) at about 293 million years, as opposed to 10,000 years as previously estimated [5]. These authors found a general agreement in the inferred branch lengths between evolutionary models for the shorter branches (inferior to 0.05 substitution per site). As expected in the phylogeny of two species of the same coronavirus genus involved in a relatively recent zoonotic transmission event, all the branches of neighbor joining and Bayesian trees were shorter than 0.03 substitutions per site, as shown in Figures 1 and 2. It can be speculated that the phylogenetic analyses presented in this study did not underestimate the date of emergence of the different clusters nor the estimation of tMRCA for $\mathrm{HCoV}-\mathrm{OC} 43$ and $\mathrm{BCoV}$. The molecular clock analysis indicated that HCoV-OC43 and BCoV strains have emerged from their tMRCA around 1890 (1859-1952), which was consistent with the data published by Vijgen et al. in 2006 [39]. The emergence of cluster E, characterized by a TQGD amino acid deletion, is estimated at around 1944 (1942-1968), and would have occurred at the same time as that of cluster A, in the 1942-1958 time range. The cluster A includes the first HCoV-OC43 strain identified in 1967 from a respiratory sample [13]. The B and C clusters would have circulated later, since the 1980s. The molecular clock results therefore suggest that, since their emergence in the late nineteenth century, the evolution of circulating HCoV-OC43 epidemic strains would have been marked by the occurrence of an insertion of four amino acids, TQDG, in the N-terminal region of the S1 attachment protein, as opposed to the occurrence of the deletion of these four amino acids. The pursuit of this study should lead to a means of determining the functional impact of this insertion, especially with regard to tropism and the clinical expression of infection by $\mathrm{HCoV}-\mathrm{OC} 43$. Note that the frequent presence of gastrointestinal symptoms in the clinical landscape of $\mathrm{HCoV}-\mathrm{OC} 43$ respiratory infections has yet to be accounted for. 
In 2014, Hu et al. studied the genetic diversity of the HCoV-OC43 circulating in Beijing, China [50]. The authors performed partial sequencing of the S1 and $\mathrm{N}$ genes (24,228-24,785 and 30,022-30,580 for $\mathrm{S} 1$ and $\mathrm{N}$ respectively, using the $\mathrm{HCoV}-\mathrm{OC} 43$ sequence associated with the accession number NC_005147 as reference) of $50 \mathrm{HCoV-OC43s} \mathrm{detected} \mathrm{in} \mathrm{the} \mathrm{respiratory} \mathrm{samples,} \mathrm{all} \mathrm{taken} \mathrm{in} 2012$. They included the sequences published by Lau et al. in their analysis in 2011, and used the nomenclature established by these same authors to identify various clusters obtained $[33,50]$. In addition to the $A$ and $B$ clusters, they identified 4 other groups, designated UNT1, UNT(B), $\mathrm{UNT}(\mathrm{C} / \mathrm{D})$, and (C/D), respectively. Several clusters were found to correspond to those previously described by Lau et al. in 2011 [33]. The UNT(B) cluster or "Untyped B" is a group close to the B cluster. The C/D cluster corresponds to the C cluster, named "C/D", due to the presence of the HK04_02 strain defined by Lau et al. as a genotype $\mathrm{D}$, referring to a recombination virus belonging to the $\mathrm{B}$ cluster on the nsp12 sequence and to the $\mathrm{C}$ cluster on the $\mathrm{S}$ and $\mathrm{N}$ sequences [33]. The UNT(C/D) cluster or "Untyped (C/D)" corresponds to a group close to the preceding cluster. Finally, the UNT1 cluster or "Untyped 1", which is close to the $\mathrm{BCoV}$ sequences included in this analysis, might correspond to the E cluster describe in the current study. In the report by Hu et al. (2014), the UNT1 cluster includes only five HCoV-OC43s (accession numbers Z32769, HC734573, CQ772300, DD266155, and Z32768) [50]. We have verified that they all have the characteristic nucleotide deletion of 12 nucleotides. This very recent report supports the results determined in the current study in that they both demonstrate a high genetic diversity of circulating $\mathrm{HCoV}-\mathrm{OC} 43 \mathrm{~s}$.

The present study highlights the difficulty of investigating intraspecific genetic variability of $\mathrm{HCoV}-\mathrm{OC} 43 \mathrm{~s}$, though the analysis of partial viral genomes (complete genes) allows the description of clear recombination patterns. Nevertheless, due to the high evolutionary potential of HCoV-OC43, analyzing complete genomes would reveal a more detailed and perhaps more complex recombination pattern. In addition, the data reported and analyzed here illustrate the dynamic diversification of $\mathrm{HCoV}-\mathrm{OC} 43$ following its emergence in human. This remarkable diversification contrasts with the relative stability of $\mathrm{BCoV}$ in cattle (unpublished data). The present study therefore draws attention to the importance of increasing our knowledge of the evolution dynamism of coronavirus in combating the permanent emergence risk that they pose in the human population, evidenced by the SARS pandemic of 2002-2003 and the recently identified MERS-coronavirus in the Middle-East.

\section{Materials and Methods}

\subsection{Clinical Samples and Positive Control}

Fifteen human respiratory specimens (upper and lower) positive for HCoV-OC43 using molecular detection, sampled from 2001 to 2013 (one to two for each year) were chosen among the specimens available at the virology department of the University Hospital of Caen. These specimens were sampled from patients suffering from acute upper or lower respiratory diseases, and were sent to our laboratory for a viral diagnosis. The detection of HCoV-OC43 was performed using an in-house real-time quantitative RT-PCR, for which the original primers may be found in Table 4. Clinical signs, age, and sex of patients were investigated and compiled in Table 3. We also used a culture supernatant of the 
prototype strains VR759 as reference and for validation of our method. This strain was obtained from the ATCC and maintained in HRT-18 cells.

Table 4. Primer and probes used for RT-PCR and full sequencing of nsp12, S, and N genes of $15 \mathrm{HCoV}-\mathrm{OC} 43 \mathrm{~s}$, and the prototype strain VR759.

\begin{tabular}{|c|c|c|c|c|c|}
\hline \multicolumn{6}{|c|}{ Real-Time RT-PCR } \\
\hline $\begin{array}{c}\text { Primer or } \\
\text { Probe Name }\end{array}$ & Polarity & Primer/Probe Sequences (5'-3') & $\begin{array}{c}\text { Gene } \\
\text { Target }\end{array}$ & Location (5'-3') & Reference \\
\hline LOC1 & fwd & GTGGTTTTGCTGTTTATGTTAAGT & $\mathrm{N}$ & 28991-29014 & this study \\
\hline LOC2 & rev & AGATATTATTTCTCAACAATGCGGT & $\mathrm{N}$ & $29092-29068$ & this study \\
\hline SLOC & fwd & HEX-AATTACCGACTGCCATCAACCCAAA-BHQ1 & $\mathrm{N}$ & 29026-29050 & this study \\
\hline \multicolumn{6}{|c|}{ RT-PCR and sequencing } \\
\hline Primer name & Polarity & Primer sequences (5'-3') & $\begin{array}{c}\text { Gene } \\
\text { target }\end{array}$ & Location (5'-3') & Reference \\
\hline OC43_P_13158F & fwd & TTGTGCAAATTACGCGGCAA & $\mathrm{RdRp}$ & $13171-13190$ & this study \\
\hline OC43_P_13896R & rev & TTCACCAATTTGTCCGCAAAC & $\mathrm{RdRp}$ & $13907-13889$ & this study \\
\hline OC43_P_13645F & fwd & GCCACACATTGTACGCAAGG & $\operatorname{RdRp}$ & $13649-13668$ & this study \\
\hline OC43_P_14511R & rev & CCGCTTGTTATAGCGGCAAC & RdRp & $14515-14496$ & this study \\
\hline OC43_P1_4375F & fwd & GTGGATACACATCGTTATCGCTT & $\operatorname{RdRp}$ & $14379-14401$ & this study \\
\hline OC43_P_15253R & rev & CCTATCGCTTTGCGAACAACA & $\mathrm{RdRp}$ & $15257-15237$ & this study \\
\hline LPW 3064F & fwd & CTGGGATGATATGTTACGCCG & $\operatorname{RdRp}$ & $15095-15115$ & Lau et al. 2011 \\
\hline LPW 2579R & rev & GTGTGTTGTGAACARAAYTCRTG & $\operatorname{RdRp}$ & $15763-15750$ & Lau et al. 2011 \\
\hline OC43_P_15276F & fwd & TGAGTGATGATGGGGTTGTGT & $\operatorname{RdRp}$ & $15577-15597$ & this study \\
\hline OC43_P_16129R & rev & TTGAGAAGAGCAGACCACGC & $\mathrm{RdRp}$ & $16133-16114$ & this study \\
\hline OC43_P_15814F & fwd & AGGAGCTGGATGTTTTGTAGATGA & $\operatorname{RdRp}$ & $15818-15841$ & this study \\
\hline LPW 1127R & rev & TGCCTTTTGCGTTTCTGC & $\operatorname{RdRp}$ & $16520-16503$ & Lau et al. 2011 \\
\hline LPW 1162F & fwd & CCYRTTTGTRTGTATGATCC & $\mathrm{S}$ & $23500-23520$ & Lau et al. 2011 \\
\hline LPW 1166R & rev & YGCATAAAAAGTACCACC & $\mathrm{S}$ & $24325-24307$ & Lau et al. 2011 \\
\hline OC43_S_23506F & fwd & TGTGTGTATGATCCGCTACCAG & $\mathrm{S}$ & $23507-23528$ & this study \\
\hline OC43_S_24384R & rev & GTGAAAGYGCCATGCCTAAA & S & $24391-24372$ & this study \\
\hline LPW 6447F & fwd & CTTCAAAGAACTATGGCATT & $\mathrm{S}$ & $24198-24217$ & Lau et al. 2011 \\
\hline LPW 6548R & rev & GACTGCAAATAGCCCAAATT & $\mathrm{S}$ & $24917-24898$ & Lau et al. 2011 \\
\hline LPW 2095F & fwd & TGATGCTGCTAAGATATATGG & $\mathrm{S}$ & $24804-24824$ & Lau et al. 2011 \\
\hline OC43_S_24569R & rev & AACCTCAACAAAAATGCCTTGG & S & $25581-25560$ & this study \\
\hline OC43_S_25372F & fwd & AGCATTTTTGGGTTGGTCTGC & $\mathrm{S}$ & $25383-25402$ & this study \\
\hline OC43_S_26126R & rev & AATCACCACAGACAAATGCAGC & S & $26137-26116$ & this study \\
\hline OC43_S_25882F & fwd & AGGTAGTGGTTACTGTGTGGA & $\mathrm{S}$ & $25893-25913$ & this study \\
\hline LPW 1178R & rev & GACACCAAGMCCATTAAT & $\mathrm{S}$ & 26652_26635 & Lau et al. 2011 \\
\hline OC43_S_26406F & fwd & TGATGTCGGTTTTTGTTGAGGC & $\mathrm{S}$ & $26418-26438$ & this study \\
\hline OC43_S_27152R & rev & CAGACCGGGACTAACCTTCG & $\mathrm{S}$ & $27162-27143$ & this study \\
\hline OC43_S_26971F & fwd & TGCAGCACAAGCTATGGAGA & $\mathrm{S}$ & $26982-27001$ & this study \\
\hline LPW 1275F & fwd & TRAAATGGCCTTGGTATGT & $\mathrm{S}$ & $27548-27566$ & Lau et al. 2011 \\
\hline LPW 1189R & rev & TKWMWAGGAACTCTACAATA & $\mathrm{S}$ & $27942-27923$ & Lau et al. 2011 \\
\hline OC43_N_28778F & fwd & TGTTAGGCCGATAATTGAGGACT & $\mathrm{N}$ & $28803-28825$ & this study \\
\hline OC43_N_28728F & fwd & AACCCAGAAACAAACACY & $\mathrm{N}$ & $28753-28769$ & this study \\
\hline OC43_N_29546R & rev & GCGGTCCTGTTCCCAGATAG & $\mathrm{N}$ & $29500-29481$ & this study \\
\hline OC43_N_29232F & fwd & CAGCAACCATCAGGAGGGAA & $\mathrm{N}$ & $29257-29276$ & this study \\
\hline
\end{tabular}


Table 4. Cont.

\begin{tabular}{cccccc}
\hline & \multicolumn{3}{c}{ RT-PCR and sequencing } \\
\hline Primer Name & Polarity & Primer Sequences (5'-3') & Gene Target & Location (5'-3') & Reference \\
\hline OC43_N_30104R & rev & AAACATCCTTCTGGGGCTG & N & $30148-30130$ & this study \\
OC43_N_29158F & fwd & CCGATCAGTCCGACCAGTTT & N & $29183-29202$ & this study \\
OC43_N_30084R & rev & TCTGCACTTTGGCCAACTCT & N & $30109-30090$ & this study \\
OC43_Nd_29879F & fwd & GAATAARCCCCGCCAGA & N & $29904-29920$ & this study \\
OC43_N_30656R & rev & CATGCTGGCTCTTTCCCTTG & N & $30675-30655$ & this study \\
LPW 1195F & fwd & GAGAGGCCCTAATCAGAA & N & $29967-29984$ & Lau et al. 2011 \\
OC43_N_30690R & rev & TTAACTTCATTCATTTACTA & N & $30715-30696$ & this study \\
\hline
\end{tabular}

\subsection{RNA Extraction and Quantitative RT-PCR}

Total nucleic acids of $300 \mu \mathrm{L}$ of clinical specimens and VR759 cultures supernatant were extracted using the QiAsymphony automate and the QIAsynphony virus/bacteria kit (Qiagen, Holden, Germany), following instructions of manufacturer.

\subsection{Sequencing of the 3 Complete Genes Nsp12, S and N}

To determine the genomic sequence of nsp12, S, and N genes, a set of 17 overlapping RT-PCR products, from 600 to 900 nucleotides, were generated. These RT-PCR products encompassed the entire three genes. Twelve primers were used to amplify and sequence the nsp12 gene, composed of 2783 nucleotides and located from nucleotides 13,317 to 16,099 (reference to VR759, NC005147). Eighteen primers were used to amplify and sequence the $\mathrm{S}$ gene composed of 4062 nucleotides. This gene is located in the last third of the HCoV-OC43 genome, between bases 23,644 and 27,729 (reference to HCoV-OC43 VR759, NC005147). Six primers were used to amplify and sequence the N gene composed of 1347 nucleotides and located at the 3' end of the HCoV-OC43 genome, from nucleotide 29,104 to 30,450 (reference to HCoV-OC43 VR759, NC005147). For both RT-PCR and sequencing reactions, primers were selected in conserved domains from an alignment of the previously published HCoV-OC43. Some of the selected primers were published by Lau in 2011, but others were designed using Primer-Blast software (http://www.ncbi.nlm.nih.gov/tools/primer-blast/) [33]. All of them were tested in silico and in vitro with the VR759 prototype strain to test their efficacy. In vitro experimentations have also been conducted using the VR759 strain to test the primer abilities to provide us with quality sequencing products. Alternative original primers were used for HCoV-OC43/FRA_EPI/Caen/2001/02, HCoV-OC43/FRA_EPI/Caen/2002/04, HCoV-OC43/FRA_EPI/Caen/2007/09, HCoV-OC43/FRA_EPI/Caen/2001/01, and HCoV-OC43/FRA_EPI/ Caen/2002/03. The sequences of the 42 primers used for RT-PCR and sequencing are given in Table 4 , associated with the corresponding targeted gene and with the fragment size. RT-PCR reactions were performed using OneStep RT-PCR System (Qiagen, Holden, Germany), from $2.5 \mu \mathrm{L}$ of nucleic acid in a final volume of $25 \mu \mathrm{L}$. The same primers were used to perform the complete bidirectional sequencing of the RT-PCR products, with a Sanger derived method. $2.5 \mu \mathrm{L}$ of each RT-PCR product were first purified by adding $1 \mu \mathrm{L}$ of ExoSAP-IT (USB corporation, Cleveland, OH, USA), and by heating it at $37{ }^{\circ} \mathrm{C}$ for $15 \mathrm{~min}$, followed by $15 \mathrm{~min}$ at $80{ }^{\circ} \mathrm{C}$ to disable enzymatic activities. Two sequencing reactions were performed for each RT-PCR product with either a forward or a reverse primer. 1 to $2 \mu \mathrm{L}$ of 
purified RT-PCR product were used in a final volume of $10 \mu \mathrm{L}$ of reaction mixture, made up with the BigDye terminator cycle sequencing kit, version 1.1 (Applied Biosystems, Foster City, CA, USA), in addition to $1 \mu \mathrm{L}$ of one of the corresponding primers. The cycling parameters for the sequencing RT-PCR were $96{ }^{\circ} \mathrm{C}$ for $1 \mathrm{~min}$, followed by 30 cycles of $96^{\circ} \mathrm{C}$ for $10 \mathrm{~s}, 60^{\circ} \mathrm{C}$ for $5 \mathrm{~s}$, and $60{ }^{\circ} \mathrm{C}$ for 4 min. The analysis of labelled products was performed by the capillary electrophoresis ABI Prism 3500 genetic analyzer (Applied Biosystems, Foster City, CA, USA), by the molecular genetics laboratory of the University Hospital of Caen. In this study, the term of molecular isolate was use to designate the sequence of $\mathrm{HCoV}-\mathrm{OC} 43$ detected directly from clinical sample.

\subsection{Bioinformatic Analysis}

Sequences were assembled in contigs corresponding to the entire nsp12, $\mathrm{S}$ or $\mathrm{N}$ genes with CodonCode Aligner Software, version 5.0.1 (CodonCode corporation, Centerville, MA, USA). Multiple sequence alignment and phylogenetic analysis were performed using MEGA software, version 6.06 (http://www.megasoftware.net). Twenty-one sequences available in GenBank were added to the alignment, including HCoV-OC43s HKU4_01 and HKU4_02 from Hong-Kong [33]; HCoV-OC43s BE03 and BE04 from Belgium [34]; HCoV-OC43s OC43/human/USA/851-15/1985, OC43/human/USA/007-11/2000, OC43/human/USA/925-1/1992, OC43/human/USA/8912-37/1989, OC43/human/USA/953-23/1995, OC43/human/USA/951-18/1995, OC43/human/USA/872-5/1987, OC43/human/USA/901-54/1990, OC43/human/USA/931-85/1993, OC43/human/USA/911-58/1991, OC43/human/USA/008-5/2000, OC43/human/USA/832-27/1983, OC43/human/USA/965-6/1996 and OC43/human/USA/971-5/1997 from the USA, recently submitted by Town et al.; and BCoV Kakewaga, Mebus, and Quebec to root the trees [36,38,51]. The accession numbers corresponding to these sequences are given in Table 3. Phylogenetic trees were constructed using the method of neighbor joining, with the substitution model of Kimura-2, implemented in MEGA6 [41,52]. These trees were used to perform the comparative analysis of our results with those of Lau et al in 2011 [33]. In order to support neighbour joining trees with a probabilistic method and to estimate divergence time between groups and subgroups, trees of $\mathrm{S}$ and $\mathrm{N}$ genes were also inferred using the same sequences and a Bayesian Markov Chain Monte Carlo method, implemented in the BEAST package, version 1.8.1 [42,53]. Inferences were calculated with the one parametric coalescent model with a constant size, under the TN93+G substitution model according to Bayesian Information Criterion (BIC) and Akaike Information Criterion (AIC) values of a model test carried out on MEGA6 software. The tMRCA was estimated using a relaxed molecular clock with an uncorrelated lognormal distribution. The length of MCMC was fixed at $3 \times 10^{-8}$ states for the both genes. The Tracer software, version 1.5, implemented in the BEAST package, was used to assess the fitness of the model used, focusing the Effective Sampling Size (ESS) data, after a burning of $10 \%$. The target trees were obtained with the maximum credibility clades, with a posterior probability limit of 0.05 . The tMRCA age for each genotype was compared for $\mathrm{S}$ and $\mathrm{N}$ genes.

\section{Acknowledgments}

This work was supported by the French Agence Nationale de la Recherche (ANR) on the behalf of EPICOREM project (Eco-Epidemiology of Coronaviruses, from Wildlife to Human: Emergence 
Threat Assessment), grant ANR-13-BSV3-0013. We thank Paul Brown for the English proofreading of the manuscript.

\section{Author Contributions}

A.V. and N.K. design the study. N.K. and W.L. performed the experiments. NK. and M.A.G. performed the analyses. N.K. conceived the manuscript with contributions from F.M., M.A.G. and A.V. All authors contributed toward the preparation, editing and proofreading of the manuscript.

\section{Conflicts of Interest}

The authors declare no conflict of interest.

\section{References}

1. Gorbalenya, A.E.; Enjuanes, L.; Ziebuhr, J.; Snijder, E.J. Nidovirales: Evolving the largest RNA virus genome. Virus Res. 2006, 117, 17-37.

2. Lauber, C.; Ziebuhr, J.; Junglen, S.; Drosten, C.; Zirkel, F.; Nga, P.T.; Morita, K.; Snijder, E.J.; Gorbalenya, A.E. Mesoniviridae: A proposed new family in the order Nidovirales formed by a single species of mosquito-borne viruses. Arch. Virol. 2012, 157, 1623-1628.

3. Adams, M.J.; Carstens, E.B. Ratification vote on taxonomic proposals to the International Committee on Taxonomy of Viruses (2012). Arch. Virol. 2012, 157, 1411-1422.

4. De Groot, R.J.; Cowley, J.A.; Enjuanes, L.; Faaberg, K.S.; Perlman, S.; Rottier, P.J.M.; Snijder, E.J.; Ziebuhr, J.; Gorbalenya, A.E. Order Nidovirales. In Virus Taxonomy: Classification and Nomenclature of Viruses: Ninth Report of the International Committee on Taxonomy of Viruses; King, A.M.Q., Adams, M.J., Carstens, E.B., Lefkowitz, E.J., Eds.; Elsevier Academic Press: San Diego, CA, USA, 2012; pp. 785-795.

5. Woo, P.C.Y.; Lau, S.K.P.; Lam, C.S.F.; Lau, C.C.Y.; Tsang, A.K.L.; Lau, J.H.N.; Bai, R.; Teng, J.L.L.; Tsang, C.C.C.; Wang, M.; et al. Discovery of seven novel mammalian and avian coronaviruses in the genus deltacoronavirus supports bat coronaviruses as the gene source of alphacoronavirus and betacoronavirus and avian coronaviruses as the gene source of gammacoronavirus and deltacoronavirus. J. Virol. 2012, 86, 3995-4008.

6. Alekseev, K.P.; Vlasova, A.N.; Jung, K.; Hasoksuz, M.; Zhang, X.; Halpin, R.; Wang, S.; Ghedin, E.; Spiro, D.; Saif, L.J. Bovine-like coronaviruses isolated from four species of captive wild ruminants are homologous to bovine coronaviruses, based on complete genomic sequences. J. Virol. 2008, 82, 12422-12431.

7. Woo, P.C.Y.; Lau, S.K.P.; Huang, Y.; Yuen, K.-Y. Coronavirus diversity, phylogeny and interspecies jumping. Exp. Biol. Med. 2009, 234, 1117-1127.

8. Ballesteros, M.L.; Sanchez, C.M.; Enjuanes, L. Two amino acid changes at the N-terminus of transmissible gastroenteritis coronavirus spike protein result in the loss of enteric tropism. Virology 1997, 227, 378-388. 
9. Chouljenko, V.N.; Lin, X.Q.; Storz, J.; Kousoulas, K.G.; Gorbalenya, A.E. Comparison of genomic and predicted amino acid sequences of respiratory and enteric bovine coronaviruses isolated from the same animal with fatal shipping pneumonia. J. Gen. Virol. 2001, 82, 2927-2933.

10. Zhang, J.; Guy, J.S.; Snijder, E.J.; Denniston, D.A.; Timoney, P.J.; Balasuriya, U.B.R. Genomic characterization of equine coronavirus. Virology 2007, 369, 92-104.

11. Fouchier, R.A.; Hartwig, N.G.; Bestebroer, T.M.; Niemeyer, B.; de Jong, J.C.; Simon, J.H.; Osterhaus, A.D. A previously undescribed coronavirus associated with respiratory disease in humans. Proc. Natl. Acad. Sci. USA 2004, 101, 6212-6216.

12. Van der Hoek, L.; Pyrc, K.; Jebbink, M.F.; Vermeulen-Oost, W.; Berkhout, R.J.; Wolthers, K.C.; Wertheim-van Dillen, P.M.; Kaandorp, J.; Spaargaren, J.; Berkhout, B. Identification of a new human coronavirus. Nat. Med. 2004, 10, 368-373.

13. McIntosh, K.; Becker, W.B.; Chanock, R.M. Growth in suckling-mouse brain of "IBV-like" viruses from patients with upper respiratory tract disease. Proc. Natl. Acad. Sci. USA 1967, 58, 2268-2273.

14. Hamre, D.; Procknow, J.J. A New virus isolated from the human respiratory tract. Exp. Biol. Med. 1966, 121, 190-193.

15. Hamre, D.; Kindig, D.A.; Mann, J. Growth and intracellular development of a new respiratory virus. J. Virol. 1967, 1, 810-816.

16. Woo, P.C.Y.; Lau, S.K.P.; Chu, C.-M.; Chan, K.-H.; Tsoi, H.-W.; Huang, Y.; Wong, B.H.L.; Poon, R.W.S.; Cai, J.J.; Luk, W.-K.; et al. Characterization and complete genome sequence of a novel coronavirus, coronavirus HKU1, from patients with pneumonia. J. Virol. 2004, 79, 884-895.

17. Van der Hoek, L.; Sure, K.; Ihorst, G.; Stang, A.; Pyrc, K.; Jebbink, M.F.; Petersen, G.; Forster, J.; Berkhout, B.; Überla, K. Croup is associated with the novel coronavirus NL63. PLoS Med. 2005, 2, e240.

18. Birch, C.J.; Clothier, H.J.; Seccull, A.; Tran, T.; Catton, M.C.; Lambert, S.B.; Druce, J.D. Human coronavirus OC43 causes influenza-like illness in residents and staff of aged-care facilities in Melbourne, Australia. Epidemiol. Infect. 2005, 133, 273-277.

19. Falsey, A.R.; Walsh, E.E.; Hayden, F.G. Rhinovirus and coronavirus infection-associated hospitalizations among older adults. J. Infect. Dis. 2002, 185, 1338-1341.

20. Lee, W.-J.; Chung, Y.-S.; Yoon, H.S.; Kang, C.; Kim, K. Prevalence and molecular epidemiology of human coronavirus HKU1 in patients with acute respiratory illness. J. Med. Virol. 2013, 85, 309-314.

21. Vabret, A.; Mourez, T.; Gouarin, S.; Petitjean, J.; Freymuth, F. An outbreak of coronavirus OC43 respiratory infection in Normandy, France. Clin. Infect. Dis. 2003, 36, 985-989.

22. Woo, P.C.Y.; Lau, S.K.; Tsoi, H.; Huang, Y.; Poon, R.W.; Chu, C.; Lee, R.A.; Luk, W.; Wong, G.K.; Wong, B.H. Clinical and molecular epidemiological features of coronavirus HKU1-associated community-acquired pneumonia. J. Infect. Dis. 2005, 192, 1898-1907.

23. Hijawi, B.; Abdallat, M.; Sayaydeh, A.; Alqasrawi, S.; Haddadin, A.; Jaarour, N.; Alsheikh, S.; Alsanouri, T. Novel coronavirus infections in Jordan, April 2012: Epidemiological findings from a retrospective investigation. East. Mediterr. Health J. 2013, 19 (Suppl. S1), S12-S18.

24. Peiris, J.S.M.; Guan, Y.; Yuen, K.Y. Severe acute respiratory syndrome. Nat. Med. 2004, 10, S88-S97. 
25. Zaki, A.M.; van Boheemen, S.; Bestebroer, T.M.; Osterhaus, A.D.M.E.; Fouchier, R.A.M. Isolation of a novel coronavirus from a man with pneumonia in Saudi Arabia. N. Engl. J. Med. 2012, 367, 1814-1820.

26. News Scan for Mar 23, 2015. Available online: http://www.cidrap.umn.edu/news-perspective/ 2015/03/news-scan-mar-23-2015 (accessed on 24 March 2015).

27. St-Jean, J.R.; Jacomy, H.; Desforges, M.; Vabret, A.; Freymuth, F.; Talbot, P.J. Human respiratory coronavirus OC43: Genetic stability and neuroinvasion. J. Virol. 2004, 78, 8824-8834.

28. Vijgen, L.; Keyaerts, E.; Moes, E.; Thoelen, I.; Wollants, E.; Lemey, P.; Vandamme, A.-M.; van Ranst, M. Complete genomic sequence of human coronavirus OC43: Molecular clock analysis suggests a relatively recent zoonotic coronavirus transmission event. J. Virol. 2005, 79, 1595-1604.

29. Smith, E.C.; Denison, M.R. Implications of altered replication fidelity on the evolution and pathogenesis of coronaviruses. Curr. Opin. Virol. 2012, 2, 519-524.

30. Steinhauer, D.A.; Domingo, E.; Holland, J.J. Lack of evidence for proofreading mechanisms associated with an RNA virus polymerase. Gene 1992, 122, 281-288.

31. Makino, S.; Keck, J.G.; Stohlman, S.A.; Lai, M.M. High-frequency RNA recombination of murine coronaviruses. J. Virol. 1986, 57, 729-737.

32. Vabret, A.; Dina, J.; Mourez, T.; Gouarin, S.; Petitjean, J.; van der Werf, S.; Freymuth, F. Inter- and intra-variant genetic heterogeneity of human coronavirus OC43 strains in France. J. Gen. Virol. 2006, 87, 3349-3353.

33. Lau, S.; Lee, P.; Tsang, A.K.L.; Yip, C.C.Y.; Tse, H.; Lee, R.A.; So, L.-Y.; Lau, Y.-L.; Chan, K.-H.; Woo, P.C.Y.; et al. Molecular epidemiology of human coronavirus OC43 reveals evolution of different genotypes over time and recent emergence of a novel genotype due to natural recombination. J. Virol. 2011, 85, 11325-11337.

34. Vijgen, L.; Keyaerts, E.; Lemey, P.; Moës, E.; Li, S.; Vandamme, A.-M.; van Ranst, M. Circulation of genetically distinct contemporary human coronavirus OC43 strains. Virology 2005, 337, 85-92.

35. Saitou, N.; Nei, M. The neighbor-joining method: A new method for reconstructing phylogenetic trees. Mol. Biol. Evol. 1987, 4, 406-425.

36. Kanno, T.; Hatama, S.; Ishihara, R.; Uchida, I. Molecular analysis of the S glycoprotein gene of bovine coronaviruses isolated in Japan from 1999 to 2006. J. Gen. Virol. 2007, 88, 1218-1224.

37. Mebus, C.A.; Stair, E.L.; Rhodes, M.B.; Twiehaus, M.J. Pathology of neonatal calf diarrhea induced by a coronavirus-like agent. Vet. Pathol. 1973, 10, 45-64.

38. Yoo, D.; Pei, Y. Full-length genomic sequence of bovine coronavirus (31 kb). Completion of the open reading frame 1a/1b sequences. Adv. Exp. Med. Biol. 2001, 494, 73-76.

39. Vijgen, L.; Keyaerts, E.; Lemey, P.; Maes, P.; van Reeth, K.; Nauwynck, H.; Pensaert, M.; van Ranst, M. Evolutionary history of the closely related group 2 coronaviruses: Porcine hemagglutinating encephalomyelitis virus, bovine coronavirus, and human coronavirus OC43. J. Virol. 2006, 80, 7270-7274.

40. Felsenstein, J. Confidence limits on phylogenies: An approach using the bootstrap. Evolution 1985, 39, 783. 
41. Tamura, K.; Stecher, G.; Peterson, D.; Filipski, A.; Kumar, S. MEGA6: Molecular evolutionary genetics analysis version 6.0. Mol. Biol. Evol. 2013, 30, 2725-2729.

42. Drummond, A.J.; Suchard, M.A.; Xie, D.; Rambaut, A. Bayesian phylogenetics with BEAUti and the BEAST 1.7. Mol. Biol. Evol. 2012, 29, 1969-1973.

43. Dijkman, R.; Jebbink, M.F.; Gaunt, E.; Rossen, J.W.A.; Templeton, K.E.; Kuijpers, T.W.; van der Hoek, L. The dominance of human coronavirus OC43 and NL63 infections in infants. J. Clin. Virol. 2012, 53, 135-139.

44. Al-Tawfiq, J.A.; Zumla, A.; Memish, Z.A. Coronaviruses: Severe acute respiratory syndrome coronavirus and Middle East respiratory syndrome coronavirus in travelers. Curr. Opin. Infect. Dis. 2014, 27, 411-417.

45. Braden, C.R.; Dowell, S.F.; Jernigan, D.B.; Hughes, J.M. Progress in global surveillance and response capacity 10 years after severe acute respiratory syndrome. Emerg. Infect. Dis. 2013, 19, 864-869.

46. Cherry, J.D.; Krogstad, P. SARS: The first pandemic of the 21st century. Pediatr. Res. 2004, 56, 1-5.

47. Dominguez, S.R.; Sims, G.E.; Wentworth, D.E.; Halpin, R.A.; Robinson, C.C.; Town, C.D.; Holmes, K.V. Genomic analysis of 16 Colorado human NL63 coronaviruses identifies a new genotype, high sequence diversity in the N-terminal domain of the spike gene and evidence of recombination. J. Gen. Virol. 2012, 93, 2387-2398.

48. Woo, P.C.Y.; Lau, S.K.P.; Yip, C.C.Y.; Huang, Y.; Tsoi, H.-W.; Chan, K.-H.; Yuen, K.-Y. Comparative analysis of 22 coronavirus HKU1 genomes reveals a novel genotype and evidence of natural recombination in coronavirus HKU1. J. Virol. 2006, 80, 7136-7145.

49. Künkel, F.; Herrler, G. Structural and functional analysis of the $\mathrm{S}$ proteins of two human coronavirus OC43 strains adapted to growth in different cells. Arch. Virol. 1996, 141, 1123-1131.

50. Hu, Q.; Lu, R.; Peng, K.; Duan, X.; Wang, Y.; Zhao, Y.; Wang, W.; Lou, Y.; Tan, W. Prevalence and genetic diversity analysis of human coronavirus OC43 among adult patients with acute respiratory infections in Beijing, 2012. PLOS ONE 2014, 9, e100781.

51. Chang, R.Y.; Hofmann, M.A.; Sethna, P.B.; Brian, D.A. A cis-acting function for the coronavirus leader in defective interfering RNA replication. J. Virol. 1994, 68, 8223-8231.

52. Kimura, M. A simple method for estimating evolutionary rates of base substitutions through comparative studies of nucleotide sequences. J. Mol. Evol. 1980, 16, 111-120.

53. Tamura, K.; Nei, M.; Kumar, S. Prospects for inferring very large phylogenies by using the neighbor-joining method. Proc. Natl. Acad. Sci. USA 2004, 101, 11030-11035.

(C) 2015 by the authors; licensee MDPI, Basel, Switzerland. This article is an open access article distributed under the terms and conditions of the Creative Commons Attribution license (http://creativecommons.org/licenses/by/4.0/). 